Chinese Journal of Organic Chemistry

\title{
溶血磷脂酰基转移机理及有效影响因子研究
}

\author{
张康逸 ${ }^{a, b} \quad$ 张丽霞 $^{b} \quad$ 屈凌波 $^{a} \quad$ 王兴国 ${ }^{c} \quad$ 刘元法 ${ }^{*, c}$ \\ ( ${ }^{a}$ 郑州大学化学系 郑州 450001) \\ ( $b$ 河南省农科院农副产品加工研究所 郑州 450002) \\ ( ${ }^{c}$ 江南大学食品学院 无锡 214122)
}

\begin{abstract}
摘要 通过定量 ${ }^{13} \mathrm{C}$ NMR 证明了磷脂酶 $\mathrm{A}_{1}$ 具有水解磷脂 $S n-1$ 位酰基脂肪酸的专一性, 证实了 $S n-2$-溶血磷脂酰胆碱 (Sn-2-lysophosphatidylcholine, $S n$-2-LPC) 的 $S n$-2 位酰基会通过酰基转移到 $S n-1$ 位生成 $S n-1$-溶血磷脂酰胆碱 (Sn-2-lysophosphatidylcholine, Sn-1-LPC)现象的存在, 从而为磷脂酶 $\mathrm{A}_{1}$ 催化磷脂酰胆碱(Phosphatidylcholine, PC)的酶解 液中存在 $S n-2-L P C, S n-1-L P C$ 和甘油磷脂酰胆碱 $(L$-alpha glycerylphosphorylcholine, GPC)的现象提供理论依据. 依据单 甘酯自动酰基转移机理，推测出在酸、碱条件下 $S n-2-\mathrm{LPC}$ 的自动酰基转移机理，并探讨溶液极性、 $\mathrm{pH}$ 、温度、底物浓 度和反应时间等影响因子对酰基转移的影响规律. 降低酰基转移的条件为：异丙醇溶剂、 $\mathrm{pH} 7 \sim 8$ 、温度 $<35{ }^{\circ} \mathrm{C}$ 、合适 的底物浓度、缩短反应时间. 本研究为合成酰基磷脂混合物, 验证磷脂酶专一性，以及解释产生混合酰基磷脂的生物合 成途径(溶血磷脂中间体)提供了数据支撑.
\end{abstract}

关键词 溶血磷脂; 酰基转移; 机理; 影响因子

\section{Mechanism and Effective Factors of Acyl Migration of Lysophospholipid}

\author{
Zhang, Kangyi $^{a, b} \quad{\text { Zhang, } \text { Lixia }^{b} \quad \text { Qu, Lingbo }^{a} \quad \text { Wang, Xingguo }^{c} \quad \text { Liu, Yuanfa }}^{*, c}$ \\ ( ${ }^{a}$ Department of Chemistry, Zhengzhou University, Zhengzhou 450001) \\ ( ${ }^{b}$ Henan Academy of Agricultural Sciences Institute of Agricultural Products Processing, Zhengzhou 450002) \\ ( ${ }^{c}$ Jiangnan University School of Food Science and Technology, Wuxi 214122)
}

\begin{abstract}
The specificity hydrolysis $S n-1$ fatty acyl of phospholipids by phospholipase $\mathrm{A}_{1}$ and the phenomenon of acyl migration in $S n$-2-lysophosphatidylcholine $\left(S n\right.$-2-LPC) were demonstrated through ${ }^{13} \mathrm{C}$ NMR, which provided fundamental basis for enzymatic reaction solution of phosphatidylcholine (PC) including $S n$-1-lysophosphatidylcholine, $S n-2-\mathrm{LPC}$, and $L$ - $\alpha$ glycerylphosphorylcholine. Based on the automatic acyl migration mechanism of monoglycerides, the automatic acyl migration mechanism of $S n$-2-LPC was speculated in the acid condition or alkali condition. The effects of reaction parameters on the acyl migration were then explored, including the polarity of the solution, $\mathrm{pH}$, temperature, substrate concentration, and reaction time. The conditions of low acyl migration were as follows: isopropanol solvent, $\mathrm{pH} 7 \sim 8$, temperature $<35^{\circ} \mathrm{C}$, defined substrate concentration, shorten reaction time. These results contributed to a data support for the synthesis of acyl mixture of phospholipids, detection of specific phospholipase $\mathrm{A}_{1}$, and the explanation of biosynthetic pathway (lysophospholipid intermediate) of the production mixed phospholipid.
\end{abstract}

Keywords lysophospholipid; acyl migration; mechanism; effective factors

溶血磷脂(lysophospholipid)是仅含有一个脂肪酰基 的磷脂，包括溶血磷脂酰胆碱(lysophosphatidylcholine, LPC)、溶血磷脂酰乙醇胺、溶血磷脂酰肌醇等 ${ }^{[1]}$. 从分 子结构上看, 溶血磷脂保留了常见磷脂的亲油和亲水性
结构，并且在很大程度上较普通磷脂有更强的亲水性、 更好的分散性，是一种很好的乳化剂、食品添加剂和抗 菌剂, 在食品、化妆品、医药、化工、航天、军事等方 面广泛应用, 极具开发价值 ${ }^{[25]}$.

*E-mail: yuanfa.liu@gmail.com

Received April 12, 2014; revised July 14, 2014; published online August 26, 2014

Project supported by the National Natural Science Foundation of China (No. 31301502), the Key Science and Technology Program of Henan Province (No. 132102110066) and the Special Foundation for Young Scientists of Henan Academy of Agricultural Sciences (No. 2013YQ25).

国家自然科学基金(No. 31301502)、河南省重大攻关(No. 132102110066)和河南省农业科学院优秀青年科技基金(No. 2013YQ25)资助项目. 
磷脂酶 $\mathrm{A}_{1}$ (Lecitase Ultra, PLA ) $_{1}$ 是一种䍨酸酯键水 解酶, 具有水解磷脂 $S n-1$ 位脂肪酸酰基的活性 ${ }^{[6]}$, 如它 能够催化磷脂酰胆碱(Phosphatidylcholine, PC)水解生成 2-酰基-溶血磷脂酰胆碱 ( $S n$-2-acyl-lysophosphatidylcholine, $S n$-2-LPC)和脂肪酸, 但 2-酰基-溶血磷脂会部 分发生酰基位移生成 1-酰基-溶血磷脂酰胆碱(Sn-1acyl-lysophosphatidylcholine, $S n-1-\mathrm{LPC}$ ). 磷脂酶 $\mathrm{A}_{1}$ 催化 水解磷脂制备溶血磷脂过程中发生的酰基转移包含磷 酰基的转移 ${ }^{[7 \sim 9]}$ 和脂肪酸酰基的转移. 酰基转移现象的 存在是测定磷脂酶专一性 ${ }^{[10 \sim 12]}$, 合成酰基磷脂混合 物 ${ }^{[13,14]}$, 以及解释产生混合酰基磷脂的生物合成途径 (溶血磷脂中间体) ${ }^{[15,16]}$ 过程中必须考虑的问题. 1920 年, Fischer $^{[17]}$ 首次发现了酰基转移的现象, 但由于异构体和 中间过渡态在分离、鉴定方面遇到技术瓶颈，阻碍了其 影响因素的探讨和转移机理的研究. 随着检测技术和脂 质化学技术的发展, 有报道用构型分析和动力学分析研 究酰基转移机理，结果说明脂质体中的电荷、空间构型、 空间电子云和熵变是影响分子内重排的重要因素 ${ }^{[18]}$

对于 $\mathrm{PLA}_{1}$ 催化水解磷脂制备溶血磷脂中酰基转移 现象存在的证实和机理还未有确定的解释. 本研究以 $\mathrm{PLA}_{1}$ 催化 PC 水解制备 LPC 为反应模型, 利用 ${ }^{13} \mathrm{C} \mathrm{NMR}$ 结合 HPLC 进行溶血磷脂酰基转移现象的研究, 并对酰 基转移的有效影响因子如溶液极性、 $\mathrm{pH}$ 、温度、底物浓 度和反应时间等进行探讨，以期为 PLA 1 专一性的验证、 酰基磷脂混合物的存在以及解释产生混合酰基磷脂的 生物合成途径提供数据支撑.

\section{1 结果与讨论}

\subsection{PLA 1 催化 PC 水解过程分析}

在 PC 初始浓度 $51.5 \mathrm{mg} / \mathrm{mL}$, 反应温度 $53{ }^{\circ} \mathrm{C}, \mathrm{PLA}_{1}$ 添加量 $28.2 \mathrm{U} / \mathrm{mL}$ 反应条件下, 利用 HPLC-ELSD 追踪
检测 $\mathrm{PLA}_{1}$ 催化水解 $\mathrm{PC}$ 的过程, 发现酶反应液是脂肪 酸、PC、甘油磷脂酰胆碱 $(L-\alpha$-glycerylphosphorylcholine, GPC), LPC 的混合物, 含量变化见图 1 .

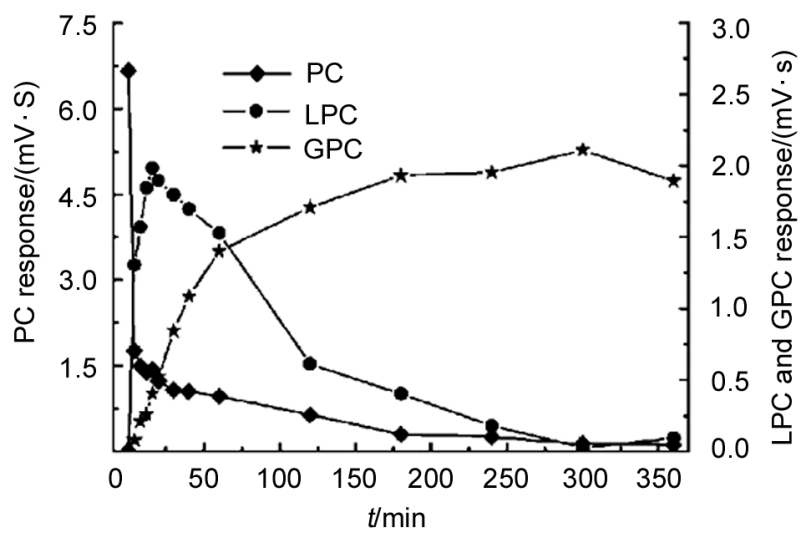

图 1 磷脂酶 $\mathrm{A}_{1}$ 水解磷脂酰胆碱过程中成分的变化规律

Figure 1 Trends of substrate and product concentrations during the hydrolysis of PC by PLA $_{1}$

从图 1 可以看出，随着 $\mathrm{PLA}_{1}$ 催化 $\mathrm{PC}$ 水解时间的延 长, $\mathrm{PC}$ 的响应值逐渐降低, 最后趋于平衡; LPC 的响应 值先快速增加，然后随着水解而响应值逐渐降低并趋向 于平衡；在反应初期，并没有检测到 GPC，随着反应时 间的延长，其响应值逐渐增大，并且生成率加快，最后 趋向于平衡. PLA 1 催化水解 PC 反应体系中 $\mathrm{LPC}$ 的变化 趋势以及 $\mathrm{GPC}$ 的存在与 $\mathrm{PLA}_{1}$ 专一性水解磷脂的 $S n-1$ 位脂肪酸生成 $S n-2-\mathrm{LPC}$ 的理论相矛盾 ${ }^{[19]}$, 因此结合图 1 中反应液中各物质的变化规律，推测 $\mathrm{PLA}_{1}$ 催化 PC 水解 制备 LPC 的过程除了 $S n-1$ 位脂肪酸水解反应外还有另 外化学现象(图 2).

$\mathrm{PLA}_{1}$ 催化 PC 水解得到 GPC 的关键步骤是 PC 的

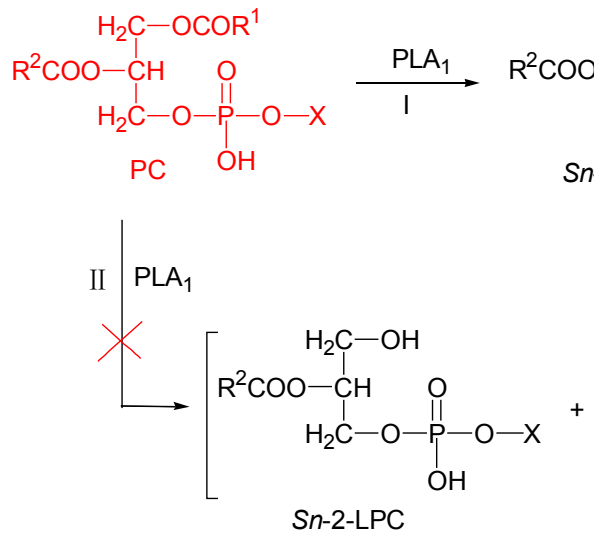<smiles>[X]OP(=O)(O)OCC(CO)C(=O)O</smiles><smiles>[X]OP(=O)(O)OCC(O)COC([R])=O</smiles><smiles>[X]O[R](=O)(O)OCC(O)CO[R]([R])=[Pt]</smiles><smiles>[X]OP(=O)(O)OCC(O)CO</smiles>

图 2 磷脂酶 $\mathrm{A}_{1}$ 水解 $\mathrm{PC}$ 示意图

Figure 2 Scheme of hydrolysis process of PC by PLA 
$S n-1$ 和 Sn-2 位脂肪酸去除方式. 假定两种情况(图 2): 一种情况是 PLA 1 是磷脂 $S n-1$ 位脂肪酸酰基水解酶, 水 解 $S n-1$ 位脂肪酸生成 $S n-2-L P C$, 生成的 $S n-2-L P C$ 发生 自动酰基转移生成新的 $S n-1-L P C, P_{1} A_{1}$ 对新生成的 $S n-1-\mathrm{LPC}$ 中 $S n-1$ 脂肪酸酰基再次水解得到 GPC(图 2 中 $\mathrm{I})$; 另一种假设情况是 $\mathrm{PLA}_{1}$ 同时水解 PC 的 $S n-1$ 和 $S n-2$ 位脂肪酸生成 $S n-2-\mathrm{LPC}$ 和 $S n-1-\mathrm{LPC}$ (图 2 中 II).

因此, 判断 PLA 1 的专一性是解释 PLA 1 水解 PC 真 实过程的基础, 也是研究自动酰基转移的依据, 为了解 释 PLA 1 催化 PC 水解制备 LPC 过程中发生的反应, 选 择水解反应的最终产物 $\mathrm{GPC}$ 为研究对象, 采用 ${ }^{13} \mathrm{C}$ NMR 进行 PLA 1 专一性验证和水溶液中 $S n-2-L P C$ 的稳 定性试验.

\section{$1.2{ }^{13} \mathrm{C} N M R$ 验证 PLA 1 的专一性}

$\mathrm{GPC}$ 是 $\mathrm{PLA}_{1}$ 催化 $\mathrm{PC}$ 水解的最终产物, 它的 ${ }^{13} \mathrm{C}$ $\mathrm{NMR}$ 谱图见图 3(c), 纯品 $\mathrm{GPC}$ 的特征碳谱为 ${ }^{13} \mathrm{C} \mathrm{NMR}$ $\left(100 \mathrm{MHz}, \mathrm{CDCl}_{3}\right), \delta_{\mathrm{C}}: 73.378(\mathrm{C}-2), 68.211(\mathrm{C}-1), 64.695$ (C-2'), 62.065 (C-3), 56.568 (C-1", 2", 3")和 42.753 (C-1').

磷脂酶 $\mathrm{A}_{2}\left(\mathrm{PLA}_{2}\right)$ 是一种催化磷脂 $S n-2$ 位脂肪酸水 解生成 $S n-1-\mathrm{LPC}$ 的专一性酶 ${ }^{[20]}$. 本研究首先用 ${ }^{13} \mathrm{C}$ $\mathrm{NMR}$ 检测 $\mathrm{PLA}_{1}$ 和 $\mathrm{PLA}_{2}$ 催化 PC 水解 $2 \mathrm{~min}$ 时的酶解 液, 通过图谱比对 [图 3(b)], 进行 $S n$-1-LPC 和 $S n$-2-LPC 的特征峰位置判定, 然后再用 ${ }^{13} \mathrm{C} N \mathrm{NM}$ 进行 PLA 1 专一 性验证. 其中 LPC-PLA $A_{1}-2$ min 表示 PLA 1 催化 PC 水解 $2 \mathrm{~min}$ 的酶解液, LPC-PLA 2 -2 min 表示 PLA 2 催化 PC 水 解 2 min 的酶解液, LPC-PLA 2 -2 min-3 h 表示 PLA 2 催化 $\mathrm{PC}$ 水解 $2 \mathrm{~min}$ 再放置 $3 \mathrm{~h}$ 的酶解液.

通过对比 $\mathrm{PLA}_{2}$ 水解 $\mathrm{PC}$ 前后酶液的 ${ }^{13} \mathrm{C}$ NMR 谱图 [图 3(b)] 发现, 在水解后酶液碳谱中(LPC-PLA 2 -2 min) 化学位移 $\delta 68.6$ 处出现了新的峰; 而 $\mathrm{PC}$ 的 ${ }^{13} \mathrm{C}$ NMR 谱 图中[图 3(b)], 化学位移 $\delta 62.8$ 处(PC 的特征吸收峰)峰 的丰度大大降低, 初步判定化学位移 $\delta 68.6$ 处为 $S n$-1-LPC 的碳谱特征吸收峰. 为了进一步验证这种判 断, 将 PLA 1 水解得到的 LPC 碳谱图(LPC-PLA $A_{1}-2 \mathrm{~min}$ ) 和 PC 的碳谱图进行对比, 发现在化学位移 $\delta 68.6$ 和 63 处得到两个新的吸收峰, 从而确定 $\delta 68.6$ 处为 $S n$-1-LPC 特征吸收峰, $\delta 63$ 处为 $S n$-2-LPC 特征吸收峰.

跟踪检测 $\mathrm{PLA}_{1}$ 水解 $\mathrm{PC}$ 的酶反应过程中 ${ }^{13} \mathrm{C} \mathrm{NMR}$ 的差别来判定 PLA $_{1}$ 的专一性, 包括对 PLA 1 催化 PC 水 解 $2 \mathrm{~min}$ 的酶解液( LPC-PLA $1-2 \mathrm{~min}$ ), $4 \mathrm{~min}$ 的酶解液 (LPC-PLA $1-4 \mathrm{~min}$ ) 和水解 $4 \mathrm{~min}$ 放置 $3 \mathrm{~h}$ 后的酶解液 (LPC-PLA $1-4$ min-3 h) 进行定量碳谱检测. 通过比对它 们的 ${ }^{13} \mathrm{C}$ NMR 谱图[图 3(b)], 发现 LPC-PLA $A_{1}-2 \min$ 和 $\mathrm{LPC}-P L A_{1}-4 \mathrm{~min}$ 酶解液的碳谱中出峰位置和峰个数都
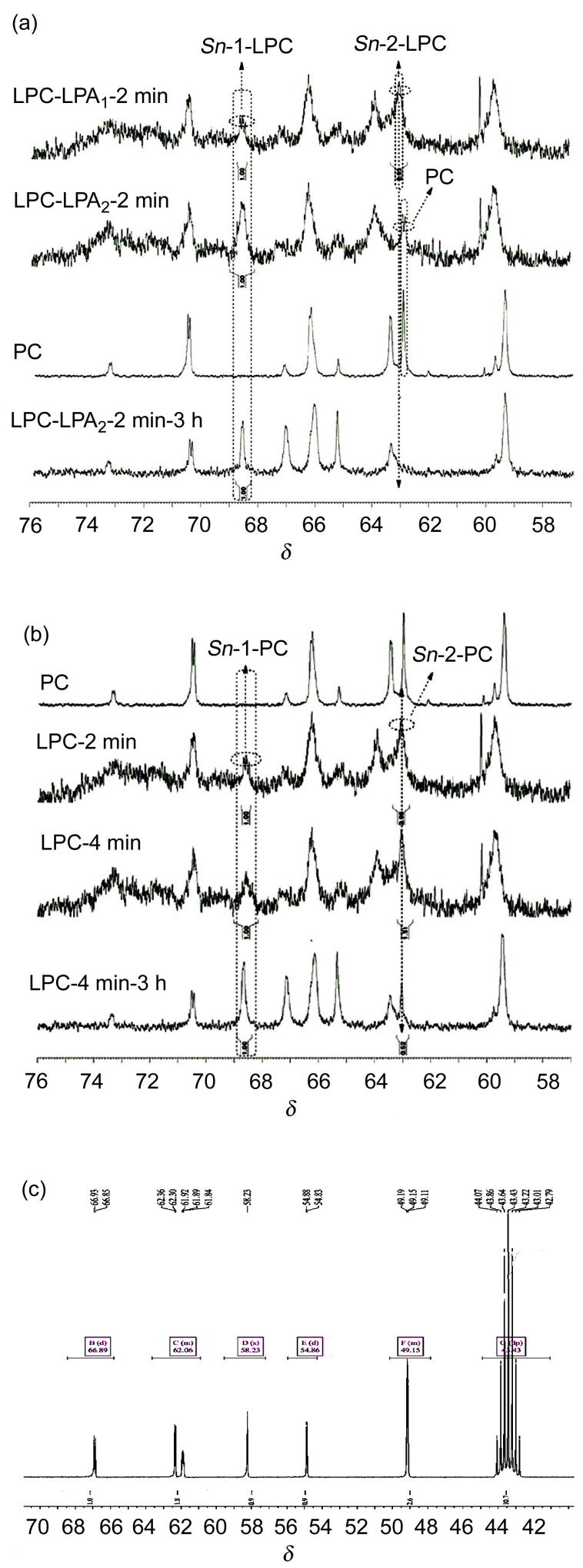

图 3 (a) $\mathrm{PLA}_{2}$ 水解 $\mathrm{PC}$ 的酶反应过程中 ${ }^{13} \mathrm{C}$ NMR 谱图、(b) $\mathrm{PLA}_{1}$ 水解 PC 的酶反应过程中 ${ }^{13} \mathrm{C} \mathrm{NMR}$ 图和(c)纯 GPC 的 ${ }^{13} \mathrm{C}$ NMR 谱图

Figure 3 (a) ${ }^{13} \mathrm{C}$ NMR profiles of hydrolysis of PC by $\mathrm{PLA}_{2}$, (b) ${ }^{13} \mathrm{C}$ NMR profiles of hydrolysis of PC by $\mathrm{PLA}_{1}$, and (c) ${ }^{13} \mathrm{C}$ NMR profiles of pure GPC 
相同, 化学位移 $\delta 63$ 和 68.6 处有两个新的吸收峰, 分别 对应 $S n$-2-LPC 和 $S n$-1-LPC 两种溶血磷脂, 不用之处在 于 $S n$-2-LPC 和 $S n$-1-LPC 丰度差异, $S n$-2-LPC 所占比例 分别为 $74.6 \%$ 和 $60.2 \%, S n-2$-LPC 和 $S n-1$-LPC 的含量比 随时间的增加而降低. 依据上面的分析, 如果 PLA $_{1}$ 不 具有专一性, 在 LPC-PLA $1-2 \mathrm{~min}$ 和 LPC-PLA ${ }_{1}-4$ min 酶 解液中应该同时存在 $S n-1-\mathrm{LPC}, S n-2-\mathrm{LPC}$ 和 GPC, 事实 恰恰相反, 在酶反应液中并没有发现 GPC, 从这一点可 以说明 PLA 1 是 $S n-1$ 专一性水解酶, 从而证明图 2 中 II 途径是不会发生的.

通过比对 LPC-PLA ${ }_{1}-4$ min 和 LPC-PLA $A_{1}-4$ min-3 h 酶反应液的 ${ }^{13} \mathrm{C}$ NMR 谱来进一步判断 $S n$-2-LPC 的稳定 性. LPC-PLA $A_{1}-4$ min 的 ${ }^{13} \mathrm{C}$ NMR 谱中, $S n-2-\mathrm{LPC}$ 的含量 为 $60.2 \%$; LPC-PLA $1-4$ min-3 $\mathrm{h}$ 中 $S n$-2-LPC 的含量降为 $34.2 \%$, 而 $S n-1-\mathrm{LPC}$ 强度相应增强, 从这个角度也可以 说明 $S n-2-\mathrm{LPC}$ 在溶液中确实存在自动酰基转移. 综合 图 1、图 3 的分析, 大胆推测 $S n-2-\mathrm{LPC}$ 自动酰基转移是 $\mathrm{PLA}_{1}$ 水解 PC 制备 GPC 的关键步骤. 在催化水解过程 中, $\mathrm{PLA}_{1}$ 先水解 PC 的 $S n-1$ 脂肪酸生成 $S n-2-\mathrm{LPC}$, $S n$-2-LPC 经过自动酰基转移生成新的 Sn-1-LPC, 新的 $S n$-1-LPC 的 $S n-1$ 位脂肪酸再次水解, 最后生成 GPC.

另外, 自动酰基转移的方向性也一直是个重要的科 学问题. 通过对比 LPC-PLA 2 -2 $\mathrm{min}$ 和 LPC-PLA $2-2$ min-3 h 酶解液的 ${ }^{13} \mathrm{C}$ NMR 谱图(图 3)发现, LPC-PLA $2-2$ min-3 h 的碳谱图在化学位移 $\delta 63$ 处并没新的吸收峰出 现, 这也证实了 LPC 的 $S n-1$ 位酰基不会转移到 $S n-2$ 位 上，这与前人报道 ${ }^{[21,22]}$ 一致.

\section{$1.3 S n-2-L P C$ 的酰基转移机理}

根据单甘酯自动酰基转移机理 ${ }^{[23]}$, 推测 $S n-2-\mathrm{LPC}$ 在酸、碱条件下的自动酰基转移机理(图 4).
在酸性条件下[图 4(a)], $\mathrm{H}^{+}$进攻羰基上的氧原子, 形成质子化的羰基氧，随后 $S n-1$ 位上羟基氧的孤对电 子进攻质子化的羰基碳正离子形成五元环中间体(图 4 中化合物 a), 断裂后生成 $S n-1-L P C$ 或者 $S n-2-L P C$. 在 碱性条件下，氢氧根负离子进攻 $S n-1$ 上的羟基氢，使羟 基氧带负电，随后进攻羰基碳正离子形成五元环中间体 (图 4 中化合物 b), 断裂后生成 $S n-1-\mathrm{LPC}$ 或者 $S n-2-\mathrm{LPC}$. 若五元环断裂生成 $S n-1-L P C$, 说明 $S n-2-L P C$ 上的脂肪 酸酰基发生了酰基转移从 $S n-2$ 位上转移到 $S n-1$ 位上.

\section{4 影响酰基转移的有效因子}

\subsection{1 溶剂体系对酰基转移的影响}

根据酰基转移的机理, 酰基转移的过程为脂肪酸酰 基的羰基碳正离子受 $S n-1$ 位上羟基氧孤对电子攻击形 成一个五元环中间体，进一步断裂形成稳定化合物的过 程. 这个过程是一种极性的变化过程, 五元环中间体的 形成是关键步骤，而溶剂体系的极性对五元环的形成产 生较大的影响. 因此本研究探讨溶液的极性对酰基转移 的影响. 在 $S n$-2-LPC 初始浓度 $0.5 \mathrm{mg} / \mathrm{mL}$, 反应温度 50 ${ }^{\circ} \mathrm{C}, \mathrm{PLA}_{1}$ 添加量 $28.2 \mathrm{U} / \mathrm{mL}$ 反应条件下, 选择极性比较 大的水、甲醇、异丙醇等溶剂, 也选择了极性比较小的 正己烷、异辛烷、乙酸乙酯等溶剂, 研究结果见表 1 .

表 1 溶剂性质对酰基转移的影响

Table 1 Effect of solvent on acyl migration

\begin{tabular}{|c|c|c|c|c|c|c|}
\hline 溶剂 & 正己烷 & 异辛烷 & 乙酸乙酯 & 异丙醇 & 甲醇 & 水 \\
\hline 溶解情况 & 不溶 & 微溶 & 微溶 & 溶解 & 溶解 & 溶解 \\
\hline 酰基转移 & - & - & - & $5.8 \%$ & $17.4 \%$ & $24.6 \%$ \\
\hline
\end{tabular}

从表 1 可以看出, 溶血磷脂 $S n-2-\mathrm{LPC}$ 在正己烷、异 辛烷、乙酸乙酯等低极性溶剂中的溶解性较差, 而在水、 甲醇、异丙醇等极性较大的溶剂体系中溶解度较好. 说 (a)<smiles>[R]C(=O)OC(CO)COP(=O)(OCC[R16]([H])([H])[SnH])OCC[N+](C)(C)C</smiles>
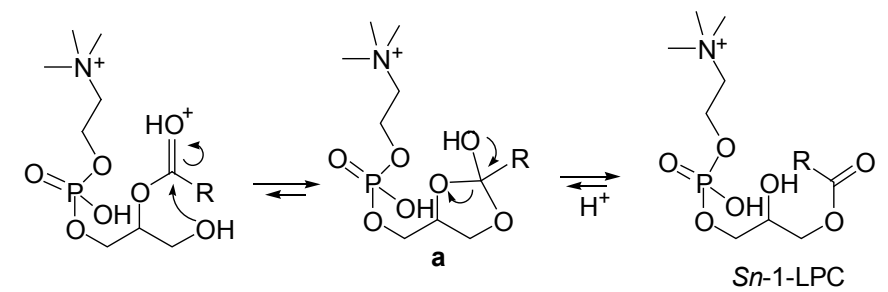

(b)

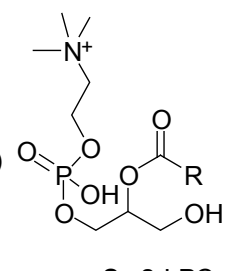

Sn-2-LPC
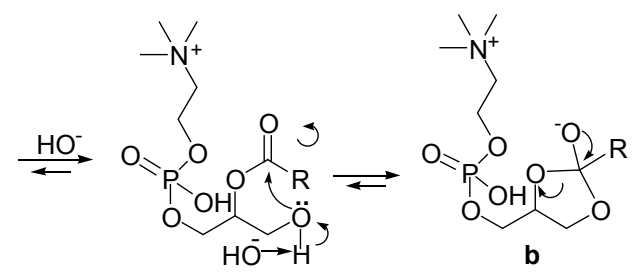
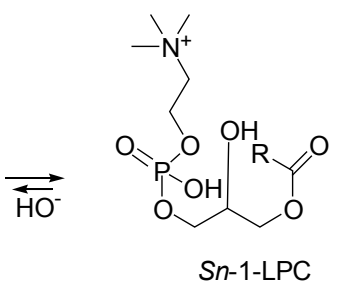

图 4 在酸碱催化条件下的酰基转移

Figure 4 Acyl migraton of Sn-2-LPC by acid and base catalysis 
明 $S n$-2-LPC 的 $S n$-1 位羟基表现出的亲水性远大于 $S n-2$ 位脂肪酸的疏水性, 根据相似相容的原理判断 $S n-2-L P C$ 呈现出较强的极性. 因此, 在极性溶剂中 $S n-2-L P C$ 溶解度较好, 并且其酰基转移的程度随溶剂 极性的增加而增加.

\subsection{2 溶液 $\mathrm{pH}$ 对酰基转移的影响}

氢正离子和氢氧根负离子是酰基转移过程中形成 五元环中间体的关键因素, 溶液的 $\mathrm{pH}$ 代表体系中氢正 离子和氢氧根负离子的浓度, 其大小对酰基转移程度有 重要影响. 在 $S n-2$-LPC 初始浓度 $0.5 \mathrm{mg} / \mathrm{mL}$, 反应温度 $50^{\circ} \mathrm{C}, \mathrm{PLA}_{1}$ 添加量 $28.2 \mathrm{U} / \mathrm{mL}$ 反应条件下, 本研究将 $S n-2-\mathrm{LPC}$ 溶于不同 $\mathrm{pH}$ 的磷酸盐缓冲溶液中, 磁力搅拌 $6 \mathrm{~h}$, 进行 $S n-2-\mathrm{LPC}$ 酰基转移程度的分析, 结果见图 5 .

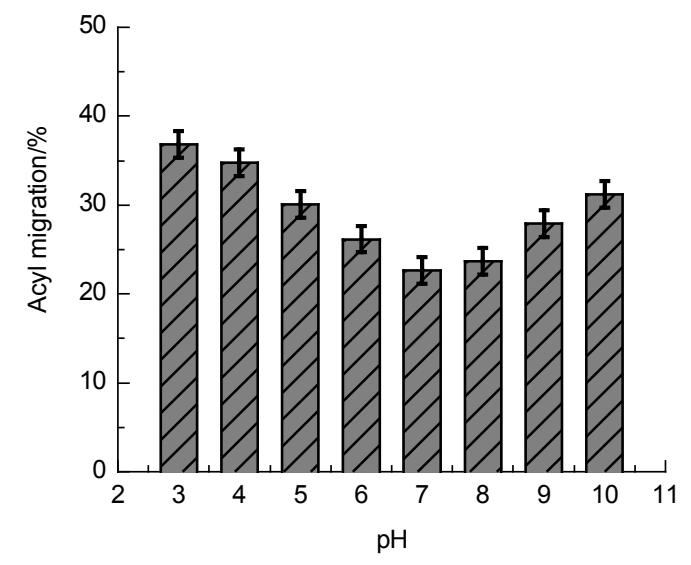

图 5 溶液 $\mathrm{pH}$ 对酰基转移的影响

Figure 5 Effect of $\mathrm{pH}$ on acyl migration

从图 5 可以看出, 在 $\mathrm{pH}<7$ 的酸性条件下, 随着 $\mathrm{pH}$ 的增加酰基转移率逐渐下降; 在 $\mathrm{pH}>7$ 的碱性条件下, 随着 $\mathrm{pH}$ 的升高酰基转移率逐渐升高; 在 $\mathrm{pH}=7$, 酰基 转移率最低(22.6\%). 因此不管是酸性条件还是碱性条 件, 都能够促进 Sn-2-LPC 酰基转移的发生. 酸性或碱性 越强, 氢正离子或者氢氧根负离子浓度越大, 越利于中 间体五元环的生成, 从而促使酰基转移的发生, 这也进 一步验证了 1.3 节中 $S n-2$-LPC 的酰基转移机理.

\subsection{3 温度对酰基转移的影响}

温度通常是影响反应的重要参数. 在 Sn-2-LPC 初 始浓度 $0.5 \mathrm{mg} / \mathrm{mL}, \mathrm{PLA}_{1}$ 添加量 $28.2 \mathrm{U} / \mathrm{mL}, \mathrm{pH} 7$ 的磷酸 盐缓冲溶液, 磁力搅拌 $6 \mathrm{~h}$ 的反应条件下, 研究温度对 Sn-2-LPC 酰基转移率的影响, 见图 6.

从图 6 可以看出, 随温度的升高酰基转移程度逐步 升高, 在 $0{ }^{\circ} \mathrm{C}$ 时 $S n-2-\mathrm{LPC}$ 的酰基转移反应几乎不发生; 在 $0 \sim 35{ }^{\circ} \mathrm{C}$, 酰基转移程度比较缓慢; $35 \sim 45{ }^{\circ} \mathrm{C}$ 的范围 内随温度的升高, 酰基转移程度快速增加; 当温度超过

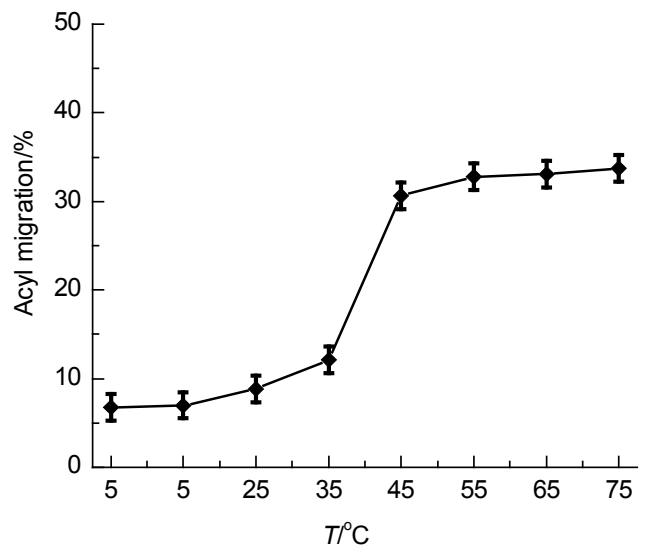

图 6 温度对酰基转移的影响

Figure 6 Effect of temperature on acyl migration

$55{ }^{\circ} \mathrm{C}$ 时, 随温度升高酰基转移程度增加不显著. 在低 温, 酰基转移率较低的原因可能为 $S n$-2-LPC 分子在低 温时的动能较低, 很难形成五元环中间体. 温度升高, $S n$-2-LPC 分子运动速率加快, 其稳定性降低, 有利于五 元环中间体的形成, 这与文献报道一致 ${ }^{[24]}$. 在温度超过 $55{ }^{\circ} \mathrm{C}$ 时反应达到平衡, 酰基转移率保持在 $33.7 \%$ 左右, 在平衡体系中 $S n$-2-LPC, $S n$-1-LPC 和 GPC 的亲水性使 得酰基转移中五元环很难形成, 所以升高温度酰基转移 率保持不变.

\subsubsection{Sn-2-LPC 浓度对酰基转移的影响}

$S n$-2-LPC 含有一个脂肪酸疏水尾，一个极性头，是 很好的乳化剂, 因此 Sn-2-LPC 浓度是影响酰基转移的 一个重要因素. 在 PLA 1 添加量 $28.2 \mathrm{U} / \mathrm{mL}, \mathrm{pH} 7$ 的磷酸 盐缓冲溶液, 反应温度 $55{ }^{\circ} \mathrm{C}$, 磁力搅拌 $6 \mathrm{~h}$ 的反应条件 下, 研究 Sn-2-LPC 浓度对酰基专一的影响(图 7).

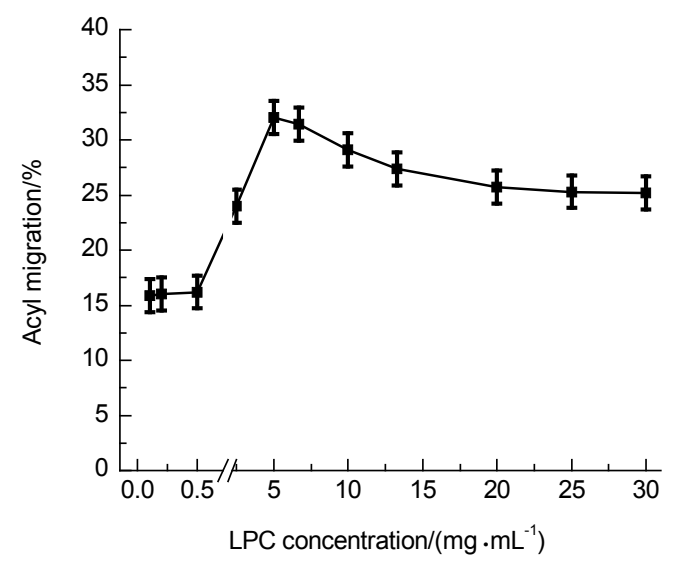

图 7 浓度对酰基转移的影响

Figure 7 Effect of concentrations on acyl migration

从图 7 中可以看出, 随着 $S n-2-\mathrm{LPC}$ 浓度的增加, 在 浓度低于 $0.5 \mathrm{mg} / \mathrm{mL}$, 酰基转移率变化不显著约 $15.8 \%$; 
浓度大于 $0.5 \mathrm{mg} / \mathrm{mL}$, 酰基转移率迅速增加; 浓度大于 5 $\mathrm{mg} / \mathrm{mL}$ ，酰基转移率反而降低; 当浓度大于 $20 \mathrm{mg} / \mathrm{mL}$, 酰基转移率维持在 25\%; Sn-2-LPC 浓度为 $5 \mathrm{mg} / \mathrm{mL}$ 时, 有最大的酰基转移率 $32 \%$. 这种现象是由多种因素共同 作用的结果, 通常情况下溶液的粘度随着底物浓度的增 加而增加, 当 $S n-2-L P C$ 浓度比较大时, 就会聚集形成小 的脂质体(图 8), 直接影响 $S n-2-\mathrm{LPC}$ 的 $S n-2$ 位脂肪酸在 空间上的舒展，从而对酰基转移中五元环中间体的形成 产生抑制作用 ${ }^{[25]}$; 当 $S n-2-\mathrm{LPC}$ 浓度比较低时, 由于 $S n$-2-LPC 的 $S n-1$ 位羟基的亲水性而分散溶于水中, $S n-2-L P C$ 分子间的距离变大, 大大降低酰基转移过程 中分子间协同相互作用，使酰基转移率较低.

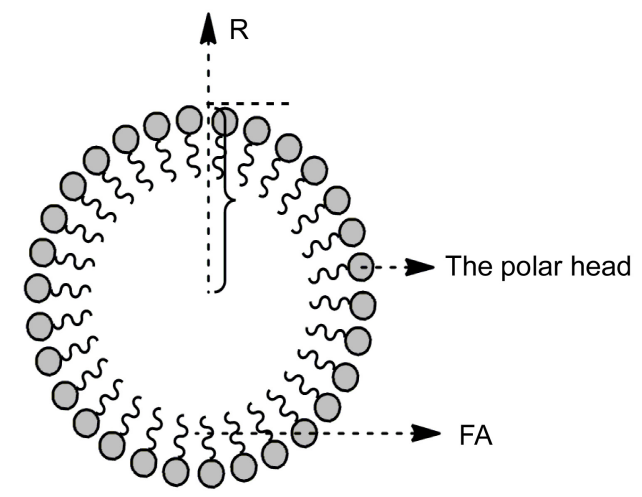

图 $8 S n$-2-LPC 的脂质体

Figure 8 Liposome of $S n$-2-LPC

\subsection{5 时间对酰基转移的影响}

酰基转移反应的过程需要一定的时间，在 $\mathrm{pH} 7$, $S n$-2-LPC 溶液浓度 $5 \mathrm{mg} / \mathrm{mL}, 55{ }^{\circ} \mathrm{C}$, 磁力搅拌下, 研究 反应时间对酰基转移的影响(图 9).

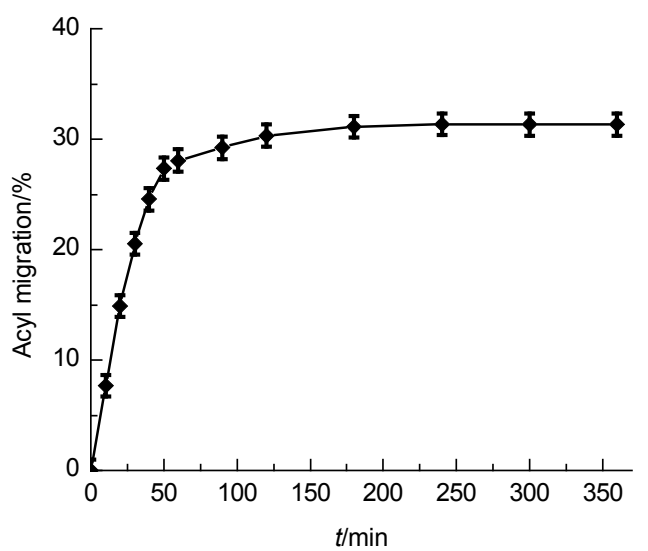

图 9 酰基转移随时间的变化规律

Figure 9 Effect of reaction time on

从图 9 中看到, 反应初始 $50 \mathrm{~min}$ 内, $S n$-2-LPC 的酰 基转移反应快速进行； $50 \sim 180 \mathrm{~min}$ 是酰基转移反应缓 慢阶段; 当时间超过 $180 \mathrm{~min}$, 酰基转移反应达到平衡,
酰基转移率维持在 $31.3 \%$.

酰基转移影响关键因子的研究结果, 可以为实际工 作中磷脂酶 $\mathrm{A}_{1}$ 催化三酰甘油酯、磷脂的酶解或者酯交 换反应提供指导，如果需要抑制酰基转移反应的发生， 可以使用低极性溶剂、 $\mathrm{pH}$ 接近中性的反应体系、尽量 低的反应温度(在保证酶活前提下)、缩短酶反应时间. 例如在 PLA 1 水解 PC 制备 GPC 的反应中, 需要利用酰 基转移这一关键步骤, 就需要使用较强极性的溶剂如水 或甲醇、酸或碱体系、稍高的反应温度、尽量延长酶反 应的时间，增加酰基转移的程度，从而实现 $\mathrm{GPC}$ 的最大 得率; 但是在 PLA 1 水解 PC 制备 $S n-2-L P C$ 溶血磷脂时, 需要控制酰基转移的发生，注意的反应条件恰恰与生成 GPC 的相反.

\section{2 结论}

${ }^{13} \mathrm{C} N M R$ 图谱比对的结果证明了 $\mathrm{PLA}_{1}$ 具有水解磷 脂 $S n-1$ 位酰基脂肪酸的专一性. 在此基础上, 利用 HPLC-RID 对 PLA 1 水解 PC 过程进行分析, 发现反应初 期体系中同时存在 $S n-1-L P C$ 和 $S n-2-L P C$ ，与 PLA 1 的 $S n-1$ 专一性水解相矛盾. 由此推测, 反应过程中存在酰 基转移现象这一关键步骤. PLA 1 水解产物和 PLA 2 水解 产物的 ${ }^{13} \mathrm{C} \mathrm{NMR}$ 图谱结果对比, 证实了 $S n-2-\mathrm{LPC}$ 的 $S n-2$ 位酰基具有向 $S n-1$ 位自动转移的特性, 而 $S n-1$ 位 酰基不会发生转移.

通过对溶液极性、 $\mathrm{pH}$ 、温度、底物浓度和反应时间 等因素对酰基转移影响的研究发现水、甲醇、异丙醇等 极性比较大的体系能溶解 Sn-2-LPC, 随着溶剂极性的 增加酰基转移也增加; 溶液的 $\mathrm{pH}$ 影响中间体五元环的 形成及其稳定性，强酸或者强碱体系中酰基转移越显 著, $\mathrm{pH}=7$ 时酰基转移最低为 $22.6 \%$; 温度对酰基转移 影响显著, 在 $35{ }^{\circ} \mathrm{C}$ 以下酰基转移比较低, 35 45 ${ }^{\circ} \mathrm{C}$ 是 酰基转移快速增加阶段, 当温度超过 $55{ }^{\circ} \mathrm{C}$ 酰基转移率 变化不显著(维持在 $33.7 \%$ ); Sn-2-LPC 底物的浓度影响 $S n-2-L P C$ 分子在溶液中的分散性和分子间的协同作用, 从而影响酰基转移率，在底物浓度 $5 \mathrm{mg} / \mathrm{mL}$, 得到 $32 \%$ 的酰基转移速率; 反应的初始阶段，酰基转移快速发生， 当超过 $180 \mathrm{~min}$ 时, 酰基转移达到平衡.

本研究以 $\mathrm{PLA}_{1}$ 催化水解 $\mathrm{PC}$ 的反应过程为模型, 研 究了 $\mathrm{PLA}_{1}$ 对 $S n-1$ 位脂肪酸酰基的专一性、反应过程中 $S n-2$ 位脂肪酸酰基转移的存在，以及影响酰基转移的关 键因子, 这些研究结果为实际应用中指导溶血磷脂或者 甘油磷脂酰胆碱的生成提供指导，同时也为磷脂酶催化 三酰甘油酯或者其他种类磷脂酶解或酯交换反应中实 现酰基转移的控制提供参考. 


\section{3 实验部分}

\section{1 仪器与试剂}

Agilent 1200 高效液相色谱仪(HPLC, 美国); 示差 检测器(RID, 美国); Waters 1525 高效液相色谱仪(美 国); Alltech3300 蒸发光散射检测器 (ELSD, 美国); Bruker AVANCE AV-500 核磁共振仪(瑞士); RE-52 旋转 蒸发仪(上海亚荣生化仪器厂); AR2140 电子精密天平 (梅特勒-托利多仪器有限公司); DL-180 A 超声波清洗 器(上海之信仪器有限公司); JJ-1B 强力恒速电力搅拌器 (金坛市金南仪器厂).

PC, GPC, Sn-1-LPC, Sn-2-LPC 和磷脂酶 A 2 , 美国 Sigma 公司; 磷脂酶 $\mathrm{A}_{1}$ (酶活力 $9500 \mathrm{U} / \mathrm{mL}$ ), 丹麦诺维 信公司; 乙腈、醋酸铵, 色谱纯, 国药集团化学试剂有限 公司; 乙醇, 色谱级, 江苏汉邦科技有限公司; 超纯水 (Millipore, $18.2 \mathrm{M} \Omega \cdot \mathrm{cm}$, TOC: $3 \mu \mathrm{g} / \mathrm{mL}$ ); 其它试剂均为 分析纯.

\section{2 实验方法}

\subsubsection{HPLC-ELSD 分析}

采用 HPLC-ELSD 分析 PC、总 LPC 和 GPC 量的变

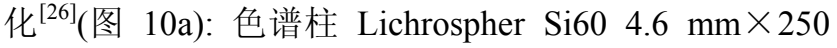
$\mathrm{mm}$; 柱温 $35{ }^{\circ} \mathrm{C}$; 二元梯度洗脱, 流动相 $\mathrm{A}$ (甲醇), 流动 相 B [ $V$ (甲醇) $: V($ 水 $)=8: 1$ ], 流速 $0.97 \mathrm{~mL} / \mathrm{min}$ (B 相的 梯度, 初始 $10 \mathrm{~min}$ 由 $40 \%$ 梯度增加到 $60 \%$ 并保持 $5 \mathrm{~min}$, 最后 $3 \mathrm{~min}$ 由 $60 \%$ 梯度降低到 $40 \%$ ). 进样量 $5 \mu \mathrm{L}$, 样品 的定性采用标样保留时间比对, 定量采用标样外标法.

\subsubsection{HPLC-RID 分析}

采用 HPLC-RID 能够将 LPC 的两类异构体 $S n$-1-LPC, $S n$-2-LPC 分离开来(图 10b), 其检测条件如 下: 色谱柱: InertSustain $\mathrm{NH}_{2} 4.6 \mathrm{~mm} \times 250 \mathrm{~mm}$, 粒径 5 $\mu \mathrm{m}$; 柱温: $25{ }^{\circ} \mathrm{C}$; 进样量: $10 \mu \mathrm{L}$. 流动相: $95 \% V($ 乙 醇) $: V$ (草酸溶液 $)=92: 8,0.45 \mu \mathrm{m}$ 有机膜抽滤并超声 脱气处理, 流速: $1 \mathrm{~mL} / \mathrm{min}$. RID 检测峰面积, 以峰面积 为纵坐标 $(Y)$ 、标样浓度为横坐标 $(X, \mathrm{mg} / \mathrm{mL})$ 进行标准曲 线的绘制.

\subsection{3 ${ }^{13} \mathrm{C} \mathrm{NMR}$}

样品的所有核磁共振谱图都采用 Bruker AVANCE-III $500 \mathrm{MHz}(11.7 \mathrm{~T})$ 型超导核磁共振波谱仪 (瑞士 Bruker 公司)在 $297 \mathrm{~K}$ 进行测定, 氞代氯仿作溶剂, 四甲基硅烷(TMS)作内标. 对于定量碳谱, 在 FT 模式下 采用反转门控 $100 \mathrm{MHz}$ 下去耦脉冲序列对样品进行信 号采集. 具体采样条件为: $30^{\circ}$ 脉冲、采样时间 $1.4 \mathrm{~s}$ 、采 样点数 $64 \mathrm{k}$ 、弛豫时间 $2 \mathrm{~s}$ 以及采样 3200 次.
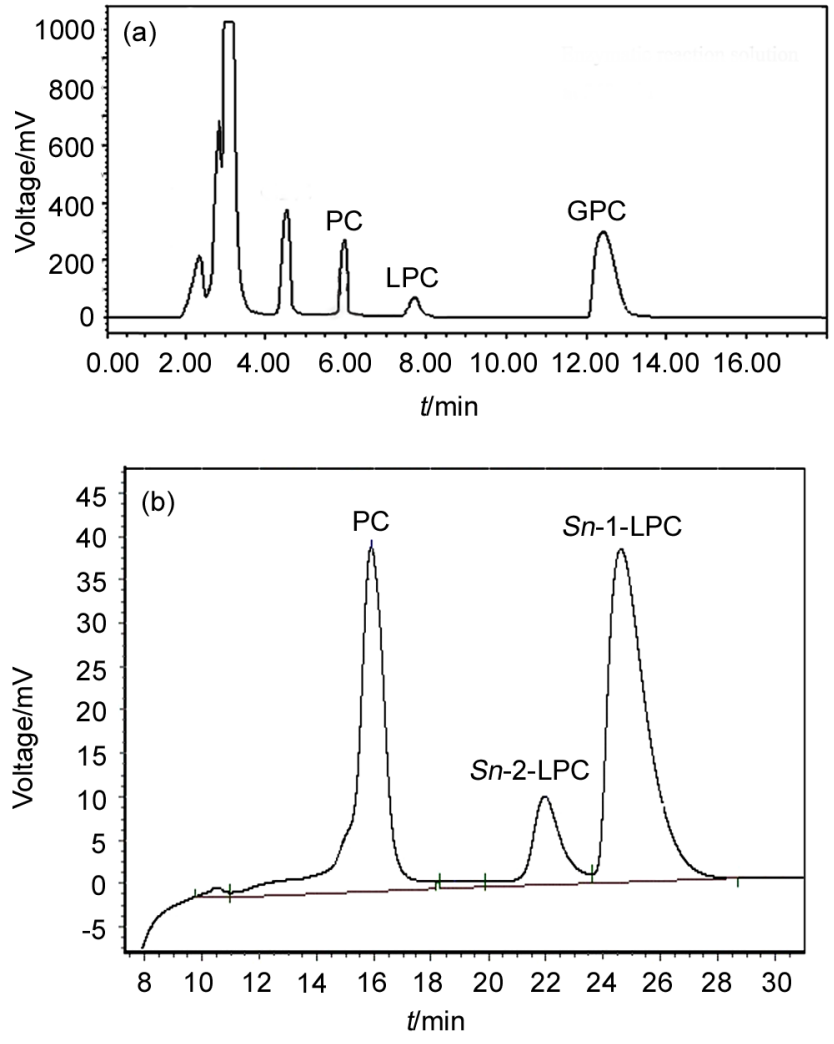

图10 (a) HPLC-ELSD和(b) HPLC-RID的HPLC分析

Figure 10 HPLC profiles of (a) HPLC-ELSD and (b) HPLC-RID

\section{References}

[1] Hu, M.-P.; Zhang, D.-J.; Zhang A.-W.; Lu, B.-X.; Li, J.-Y.; Huai, B.-D. China Oils Fats 2012, 37(11), 61 (in Chinese). (胡铭畔, 张东杰, 张爱武, 鹿保金金, 李靖元, 怀宝东, 中国油脂, 2012, 37(11), 61.)

[2] Peng, L.-F.; Xu, X.-B.; Mu, H.-L.; Hoy, C.; Adler-Nissen, J. Enzyme Microb. Technol. 2002, 31(4), 523.

[3] Saubhi, S.; Wang, Z.; Russell, T.; Xu. Y. Semin. Cell Dev. Biol. 2004, 15(5), 503.

[4] Tigyi, G. Biochim. Biophys. Acta, Mol. Cell Biol. Lipids 2013, $1831,1$.

[5] Raynal, P.; Montagner, A.; Dance, M.; Yart, A. Pathol. Biol. 2005, 53(1), 57.

[6] Slizyte, R.; Rustad, T.; Storro, I. Process Biochem. 2005, 40, 3680.

[7] Kiełbowicz, G.; Gładkowski, W.; Chojnacka, A.; Wawrzeńczyk, C. Food Chem. 2012, 135(4), 2542.

[8] Ponpipom, M. M.; Bugianesi, R. L. J. Lipid Res. 1980, 21(1), 136.

[9] Larrabee, A. L. Biochemistry 1979, 18(5), 3321.

[10] Liu, N.; Wang, Y.; Zhao, Q.-Z.; Cui, C.; Fu, M.; Zhao, M. Food Chem. 2012, 134(1), 301.

[11] Kiełbowicz, G.; Smuga, D.; Gładkowski, W.; Chojnacka, A.; Wawrzeńczyk, C. Talanta 2012, 94, 22.

[12] Dos Santos, J. C. S.; Garcia-Galan, C.; Rodrigues, R. C.; de Sant Ana, H. B.; Goncalves, L. R. B.; Fermandez-Lafuente, R. Enzyme Microb. Technol. 2014, 60, 1.

[13] Gupta, C. M.; Radhakrishnan, R.; Khorana, H. G. Proc. Natl. Acad. Sci. U. S. A. 1977, 74(10), 4315.

[14] Warner, T. G.; Benson, A. A. J. Lipid Res. 1977, 18(4), 548.

[15] Van Den Bosch, H.; Van Golde, L. M. G.; Slotboom, A. J.; Van 
Deenen, L. L. M. Biochim. Biophys. Acta 1968, 152(4), 694.

[16] Homma, H.; Nishijima, M.; Kobayashi, T.; Okuyama, H.; Nojima, S. Biochim. Biophys. Acta 1981, 663(1), 1.

[17] Fischer, E. Bet. Dtsch. Chem. Ges. 1920, 53(9), 1621.

[18] Serdarevich, B. J. Am. Oil Chem. Soc. 1967, 44(7), 381.

[19] Richmond, G. S.; Smith, T. K. Int. J. Mol. Sci. 2011, 12, 588.

[20] Ekroos, K.; Ejsing, C. S.; Bahr, U.; Karas, M.; Simons, K.; Shevchenko, A. J. Lipid Res. 2003, 44(8), 2182.

[21] Vikbjerg, A. F.; Mu, H.; Xu, X. J. Biotechnol. 2007, 128(3), 545.
[22] Poisson, L.; Devos, M.; Godet, S.; Ergan, F.; Pencreaćh, G. Biotechnol. Lett. 2009, 31(5), 743.

[23] Pliickthun, A.; Dennis, E. A. Biochemistry 1982, $21(8), 1743$.

[24] Yang, T.; Fruekilde, M. B.; Xu, X. Food. Chem. 2005, 92(1), 101.

[25] Kodali, D. R.; Tercyak, A.; Fahey, D. A.; Small, D. M. Chem. Phys. Lipids 1990, 52(3 4), 163.

[26] Zhang, K. Y.; Liu, Y. F.; Wang, X. G. Eur. J. Lipid Sci. Technol. 2012, 114, 1254. 